\title{
Randomized Trial of Personalized Breast Density and Breast Cancer Risk Notification
}

\author{
Jennifer S. Haas, MD, MSc ${ }^{7}$, Catherine S. Giess, MD², Kimberly A. Harris, MM', Julia Ansolabehere?', \\ and Celia P. Kaplan, DrPh, $M A^{3}$
}

'Division of General Medicine and Primary Care, Department of Medicine, Brigham and Women's Hospital, Boston, MA, USA; 'Department of Radiology, Division of Breast Imaging, Brigham and Women's Hospital, Boston, MA, USA; ${ }^{3}$ Division of General Internal Medicine, Department of Medicine, University of California, San Francisco, San Francisco, CA, USA.

BACKGROUND: Despite widespread implementation of mammographic breast density (MBD) notification laws, the impact of these laws on knowledge of MBD and knowledge of breast cancer risk is limited by the lack of tools to promote informed decision-making in practice.

OBJECTIVE: To develop and evaluate whether brief, personalized informational videos following a normal mammogram in addition to a legislatively required letter about MBD result can improve knowledge of MBD and breast cancer risk compared to standard care (i.e., legislatively required letter about MBD included with the mammogram result). DESIGN/PARTICIPANTS: Prospective randomized controlled trial of English-speaking women, age 40-74 years, without prior history of breast cancer, receiving a screening mammogram with a normal or benign finding (intervention group $n=235$, control group $n=224$ ). Intervention: brief (3-5 min) video, personalized to a woman's MBD result and breast cancer risk.

MAIN MEASURES: Primary outcomes were a woman's knowledge of her MBD and risk of breast cancer. Secondary outcomes included whether a woman reported that she discussed the results of her mammogram with her primary care provider (PCP).

KEY RESULTS: Relative to women in the control arm, women in the intervention arm had greater improvement in their knowledge of both their personal MBD (intervention pre/post 39.2\%/ $77.5 \%$; control pre/post $36.2 \%$ / $37.5 \%$; odds ratio (OR) 5.34 for change for intervention vs. control, 95\% confidence interval (CI) 3.87-7.36; $p<0.001$ ) and risk of breast cancer (intervention pre/ post: $66.8 \% / 74.0 \%$; control pre/post 67.9\%/ 65.2\%; OR $1.42,95 \%$ confidence interval (CI) $1.09-1.84 ; p=0.01$ ). Women in the intervention group were more likely than those in the control group to report discussing the results of their mammogram with their PCP $(p=0.05)$.

CONCLUSIONS: Brief, personalized videos following mammography can improve knowledge of MBD and personal risk of breast cancer compared to a legislatively mandated informational letter.

Electronic supplementary material The online version of this article (https://doi.org/10.1007/s11606-018-4622-4) contains supplementary material, which is available to authorized users.

Received February 6, 2018

Revised June 15, 2018

Accepted July 27, 2018

Published online August 8, 2018
Trial Registration

Clinicaltrials.gov (NCT02986360)

KEY WORDS: mammography; breast density; breast cancer risk; patient education.

J Gen Intern Med 34(4):591-7

DOI: $10.1007 / \mathrm{s} 11606-018-4622-4$

(c) Society of General Internal Medicine 2018

\section{BACKGROUND}

Breast cancer is the most common cancer in women, and mammography remains the cornerstone of breast cancer screening. Yet mammography is an imperfect test with both false-positive and false-negative results. ${ }^{1}$ Women with higher mammographic breast density (MBD) have a greater risk of breast cancer, ${ }^{2}$ and MBD can "mask" cancer on mammography, lowering the sensitivity. ${ }^{3,} 4$ Motivated by the belief that the role of MBD on risk and the problem of masking is underappreciated, patient advocacy groups have worked to pass state and national legislation to inform women about their breast density. ${ }^{5}$ As of December 2017, MBD notification laws have been enacted in 32 states, are advancing in 9 additional states, and federal legislation has been introduced; the first legislation was enacted in Connecticut in 2009. ${ }^{5}$ Massachusetts required MBD notification as of January 1, 2015. The legislation requires notification that a patient's mammogram shows dense breast tissue; dense breast tissue is common and not abnormal, but may increase the risk of breast cancer; dense breast tissue can make it difficult to find cancer on a mammogram, and sometimes, additional testing is needed for reliable breast screening; that additional screening may be advisable and that a patient should discuss the results of the mammogram with the patient's referring physician or primary care physician; the patient's right to discuss the results of the mammogram with the interpreting radiologist or physician; a report of the mammogram has been sent to the referring physician and will become part of the patient's medical record. ${ }^{6}$ Several states also require a woman to be notified that she may benefit from additional screening tests, such as breast ultrasound, and a few states mandate that private insurers cover additional screening tests for women with dense breast 
tissue. Modeling suggests that at least 28 million women aged $40-74$ (43\% of women in this age group) have higher levels of MBD and could be affected by such legislation. ${ }^{7}$

While notification laws have the well-intentioned goal of informing women about their MBD, they promote a laser focus on MDB, and downplay other important risk factors for breast cancer (e.g., age, family history), therefore limiting a broader understanding of risk. While many states specify the exact language for MBD notification, most of this language exceeds recommended readability levels. ${ }^{8}$ Few studies following the implementation of legislation suggest that a minority of women know their MBD, and that there is lack of knowledge of the effect of MBD on breast cancer risk and detection. ${ }^{9}$ Tools are needed to put MBD in the context of overall risk so that patients and providers can have more meaningful discussion. ${ }^{10}$

The objective of this study was to develop and evaluate whether brief, personalized informational videos following a normal mammogram result can improve knowledge of breast cancer risk and MBD compared to standard care (i.e., legislatively required language about MBD included with the result of the mammogram).

\section{METHODS}

\section{Overview}

INFORMD (INFORming patients after Mammography about Density) was a prospective randomized controlled trial with 1:1 batched, sequential allocation to assess whether viewing a brief, personalized informational video after a routine screening mammogram would improve women's knowledge of her breast cancer risk and MBD compared to the required breast density notification letter used in radiology practice (online Appendix includes the notification language used during the study period). Of note, Brigham and Women's Hospital sends density information to all women, not just those who have dense breast tissue as required by law. The protocol was reviewed and approved by the Institutional Review Board (IRB) of Partners Healthcare and registered at Clinicaltrials. gov (NCT02986360). There were no important changes to the study design after trial commencement.

\section{Setting and Eligibility}

Eligible patients were English-speaking women, age 4074 years, who had received a bilateral screening mammogram with a normal or benign finding (i.e., Breast ImagingReporting and Data System (BIRADS) 1 or 2) at Brigham and Women's Hospital and were receiving the results of this mammogram. Patients who did not have an email address on record in the electronic health record (EHR) were excluded, or who had a prior diagnosis of breast cancer or ductal carcinoma in situ (DCIS) documented in the EHR or by self-report.

\section{Randomization and Study Flow}

Between May 1 and October 6, 2017, eligible women were sent a letter requesting their participation in the study along with their mammogram result letter. The recruitment letter included opt-out information. If a woman did not opt-out within 2 weeks of receiving a letter, she was randomized and sent an email with a link to an online survey via Research Electronic Data Capture (REDCap). Randomization was done at the individual level, using Excel, in weekly batches. Women did not know their randomization status until they had completed the baseline assessment. Women received up to three additional reminder emails, scheduled 5 days apart. Participants who did not respond to the emails received up to two phone attempts by research staff within the following 2 weeks.

\section{Baseline Assessment}

For women in both the intervention and control arms, a baseline survey collected risk factors for breast cancer, knowledge of personal breast cancer risk (Compared with other women your age, do you think your chances of getting breast cancer are? Higher, The same, Lower, or Not sure) and MBD (Do you know your breast density? No, Yes, or Not Sure; if yes, What is your breast density? Almost entirely fatty, Scattered density, Heterogeneously dense, Extremely dense, or Not sure).

\section{Intervention}

Following the baseline survey, women in the intervention arm could view their personalized video based on their calculated breast cancer risk and MBD result. We developed four informational Vidscrips (https://www.vidscrip.com), brief (3$5 \mathrm{~min}$ ) informational videos, for women to receive following their mammogram result that varied based on a woman's breast cancer risk (high vs. average as defined below) and density result (higher vs. lower). These Vidscrips were personalized based on each woman's breast cancer risk and MBD result. The Vidscrips presented an overview of breast density, how it is measured, a woman's personal MBD, an overview of risk factors for breast cancer, a woman's personal risk for breast cancer, and personalized recommendations for screening based on risk and MBD. Breast cancer risk was calculated using the Breast Cancer Surveillance System (BCSC) model which includes age, race/ethnicity, family history of breast cancer, history of prior biopsies, and BIRADS density. ${ }^{11-13}$ This model has better prediction of breast cancer risk than other risk models and integrates density. ${ }^{12} \mathrm{MBD}$ is determined by the relative amounts of fat and connective and epithelial tissue that appear on a mammogram. It is typically defined by a qualitative description (BIRADS density "A" (almost entirely fatty), "B" (scattered areas of fibroglandular density), "C" (heterogeneously dense), or "D" (extremely dense)). 
Breast cancer risk was categorized as "high" if a woman's risk was in the top $5 \%$ of the population risk observed in the BCSC by age. ${ }^{14}$ Other women were categorized as "average" risk. MBD was categorized has higher ( $\mathrm{C}$ or $\mathrm{D})$ vs. lower (A or b).

\section{Outcome Assessment}

Two weeks following the completion of the baseline survey, participants in both the control and intervention arms were emailed an outcome assessment survey with a reminder schedule similar to that described for the baseline survey. Following the outcome assessment survey, women in the control arm received their personalized video described below to view if they wanted. Women in both study arms who completed both the baseline and outcome assessment surveys received a \$25 gift card in appreciation of their time. Outcome assessment did not change over the course of the trial.

Our primary outcomes were a woman's knowledge of her (1) MBD and (2) calculated risk of breast cancer at the time of the outcome assessment survey. Secondary outcomes included whether a woman reported that she discussed the results of her mammogram with her PCP by the time of the outcome assessment survey and, if the results were discussed, specifically

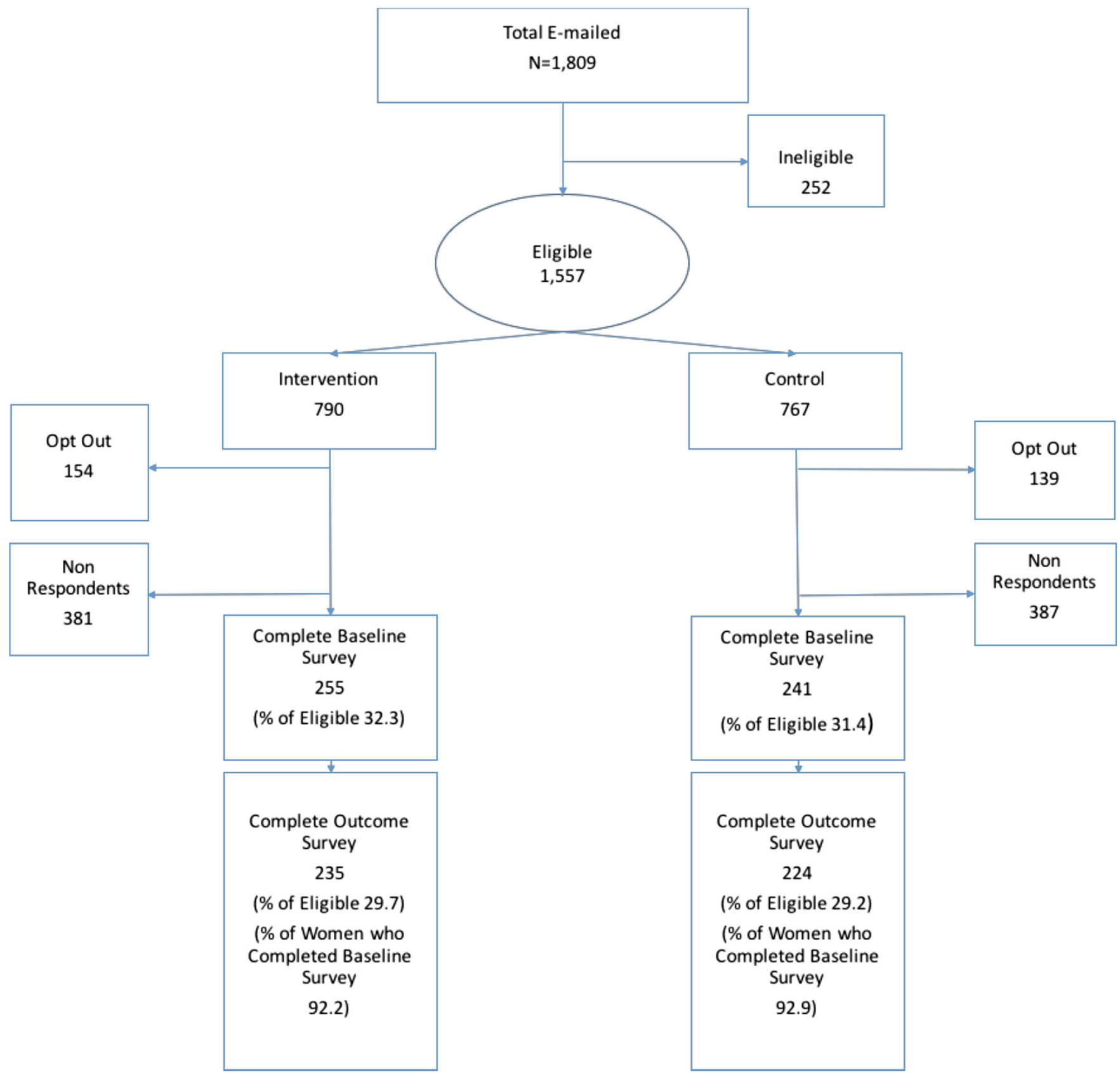

Figure 1 Eligible patients were English-speaking women, age 40-74 years, who had received a bilateral screening mammogram with a normal or benign finding (i.e., Breast Imaging-Reporting and Data System (BIRADS) 1 or 2) at Brigham and Women's Hospital and were receiving the results of this mammogram. Patients who did not have an email address on record in the electronic health record (EHR) were excluded, or who had a prior diagnosis of breast cancer or ductal carcinoma in situ (DCIS) documented in the EHR or by self-report. 
whether they discussed whether a woman should receive any additional screening tests, including ultrasound and breast magnetic resonance imaging (MRI). Within the intervention arm, we also examined whether the Vidscrip was opened, which modules were viewed, and satisfaction with the content.

\section{Data Analysis}

Sample size was selected to ensure adequate power over a range of possible baseline rates of knowledge (30-60\%) and a clinically meaningful absolute improvement of $10 \%$ for the intervention group relative to the control group. We anticipated that $25 \%$ of women who were approached would respond to the baseline assessment and that $85 \%$ of these women would complete the outcome assessment. Our goal was to have 300 women complete the outcome assessment. We had a higher than anticipated response to both the baseline and outcome assessments (Fig. 1); because of this, we increased the overall recruitment number to 500 so that we would have more high-risk women to better enable us to examine this subgroup; the study was stopped with the mailing batch that would achieve this sample size.

We compared the characteristics of respondents vs. nonrespondents and characteristics of participants in the intervention vs. control arms using Wilcoxon tests and chi-square tests. In addition to examining the overall effect of the intervention on the outcomes, we explored the effect of the intervention in subgroups based on risk and breast density. Repeated measures logistic regression was used to assess whether the change in outcomes between the baseline and outcome assessment surveys was different for the intervention vs. control arms. Analysis was based on original group assignment.

\section{RESULTS}

\section{Recruitment and Retention}

During the enrollment period, we identified 1809 women as potentially eligible based on age and mammogram result and whether an email address was listed in the EHR (Fig. 1). Of these, 252 were found to be ineligible (e.g., prior history of breast cancer). Of the 790 eligible women in the intervention arm, 255 (32.3\%) completed the baseline survey, and of these, 235 (92.2\% of baseline completers) completed the outcome assessment survey. Of the 767 eligible women in the control arm, 241(31.4\%) completed the baseline survey, and of these, 224 (92.9\% of baseline completers) completed the outcome assessment survey.

\section{Study Population}

Compared to women who did not participate, participants were more likely to be documented in the EHR as white (79.6\% vs. $64.0 \%, p<0.001)$, and a college graduate or more
(63.5\% vs. $49.6 \%, p<0.001)$. There were no differences by age or health insurance.

The median age of participants was 57 years (Table 1). Most participants self-reported that they were white, had graduated from college, and were privately insured. Nine percent of women were at higher calculated risk of breast cancer and $50.1 \%$ had higher MBD. Twenty-two percent of women perceived that they were at higher than average risk of breast cancer. Most women did not know their breast density, and of those who did, more thought that they had higher vs. lower MBD. Twenty percent of women reported ever discussing their personal MBD with their PCP prior to enrollment. There were no differences in demographic or clinical characteristics between women in the intervention and control arms.

\section{Effect of the Intervention on Ability to Correctly Identify Breast Cancer Risk and Density}

At baseline, $66.8 \%$ and $67.9 \%$ of women in the intervention and control groups respectively could correctly identify their overall

Table 1 Description of Study Participants

\begin{tabular}{|c|c|c|c|}
\hline & Intervention & Control & $p$ value* \\
\hline$N$ & 235 & 224 & \\
\hline Median age (range), years & $57(40-74)$ & $58(40-74)$ & .82 \\
\hline $\begin{array}{l}\text { Self-reported } \\
\text { race/ethnicity }\end{array}$ & & & .91 \\
\hline Hispanic & $14(6.0 \%)$ & $11(4.9 \%)$ & \\
\hline White & $192(81.7)$ & $184(82.1)$ & \\
\hline Black & $15(6.4)$ & $17(7.6)$ & \\
\hline Other/missing & $14(6.0)$ & $12(5.4)$ & \\
\hline Self-reported education & & & .46 \\
\hline High school or less & $17(7.2)$ & $10(4.5)$ & \\
\hline $\begin{array}{l}\text { Some college or technical } \\
\text { school }\end{array}$ & $30(12.8)$ & $36(16.1)$ & \\
\hline College graduate or more & $185(78.7)$ & $174(77.7)$ & \\
\hline Other/missing & $3(1.3)$ & $4(1.8)$ & \\
\hline Health insurance & & & .80 \\
\hline Medicare & 44 (18.7) & $42(18.8)$ & \\
\hline Medicaid & $8(3.4)$ & $7(3.1)$ & \\
\hline Private & $182(77.5)$ & $175(78.1)$ & \\
\hline Self-pay/uninsured & $1(0.4)$ & 0 & \\
\hline $\begin{array}{l}\text { Married or living with } \\
\text { partner }\end{array}$ & $152(64.7)$ & 137 (61.6) & .44 \\
\hline $\begin{array}{l}\text { Calculated breast cancer } \\
\text { risk }\end{array}$ & & & .45 \\
\hline Average & $211(89.8)$ & $206(92.0)$ & \\
\hline High & $24(10.2)$ & $18(8.0)$ & \\
\hline $\begin{array}{l}\text { Measured breast density } \\
\text { on mammogram }\end{array}$ & & & .08 \\
\hline Lower & $108(46.0)$ & $121(54.0)$ & \\
\hline Higher & $127(54.0)$ & $103(46.0)$ & \\
\hline $\begin{array}{l}\text { Perceived risk of breast } \\
\text { cancer }\end{array}$ & & & .96 \\
\hline Average & $150(63.8)$ & $145(64.7)$ & \\
\hline High & $54(23.0)$ & 49 (21.9) & \\
\hline Not sure & 30 (13.4) & $31(13.2)$ & \\
\hline Perceived breast density & & & .07 \\
\hline Lower & $22(9.4)$ & $35(15.6)$ & \\
\hline Higher & $85(36.2)$ & $65(29.0)$ & \\
\hline Not sure & $128(54.5)$ & $124(55.4)$ & \\
\hline $\begin{array}{l}\text { Ever discussed personal } \\
\text { breast density with your } \\
\text { primary care physician }\end{array}$ & $50(21.3)$ & $42(18.8)$ & .50 \\
\hline
\end{tabular}

*p value for median age calculated using Wilcoxon's test. All other $p$ values calculated using chi-square test 


\section{Breast Cancer Risk}

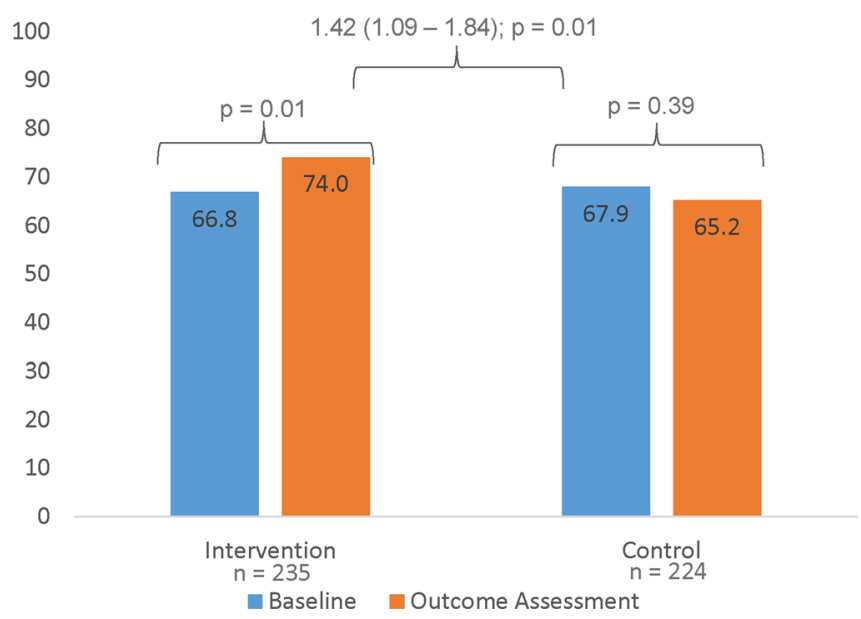

Breast Density

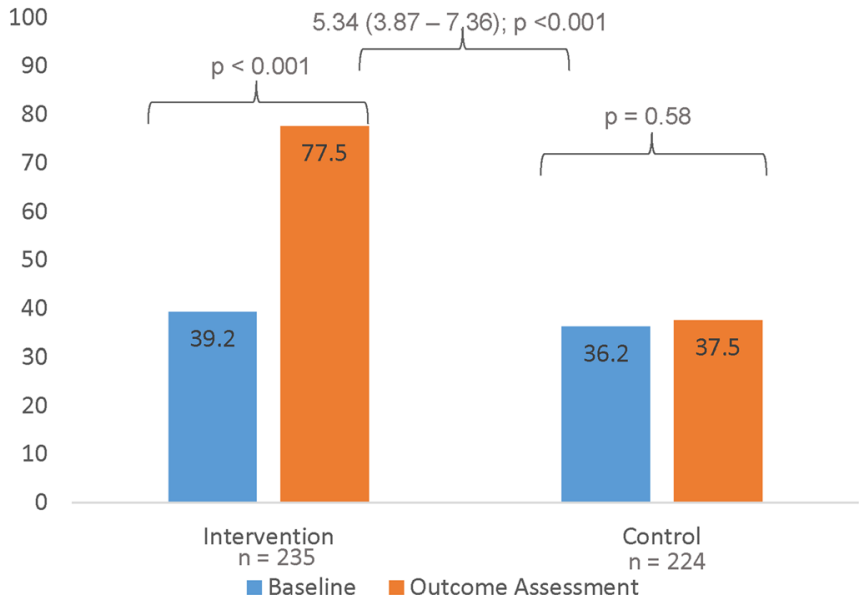

Figure 2 Effect of the intervention on knowledge of calculated breast cancer risk and measured breast density.

risk of breast cancer (Fig. 2). At outcome assessment, this increased to $74.0 \%$ for women in the intervention arm, but did not change for women in the control arm. At baseline, 39.2\% and $36.2 \%$ of women in the intervention and control groups respectively could correctly identify their MBD. At outcome assessment, this increased to $77.5 \%$ for women in the intervention arm, but did not change for women in the control arm. The intervention was significantly associated with improvement in understanding of personal risk of both breast cancer (odds ratio (OR) $1.42,95 \%$ confidence interval (CI) $1.09-1.84 ; p=0.01$ ) and MBD (OR 5.34, 95\% CI 3.87-7.36; $p<0.001)$. The effect of the intervention was significant across subgroups based on breast cancer risk and MBD except among the subgroup of women at high risk of breast cancer (Fig. 3).

\section{Discussion of Mammogram Results with PCP}

At the time of the outcome assessment survey, only $33.5 \%$ of women in the intervention group and $25.5 \%$ of women in the control group had discussed the results of their mammogram with the PCP $(p=0.05$; Table 2). Of those who discussed their result with their PCP, $35.9 \%$ of women in the intervention arm and $21.4 \%$ of those in the control arm reported that they had discussed getting additional imaging $(p=0.07)$; in the intervention arm, $18.0 \%$ discussed getting supplemental ultrasound and $14.1 \%$ discussed getting supplemental MRI vs. $5.4 \%(p=0.03)$ and $12.5 \%(p=0.79)$ respectively in the control arm.

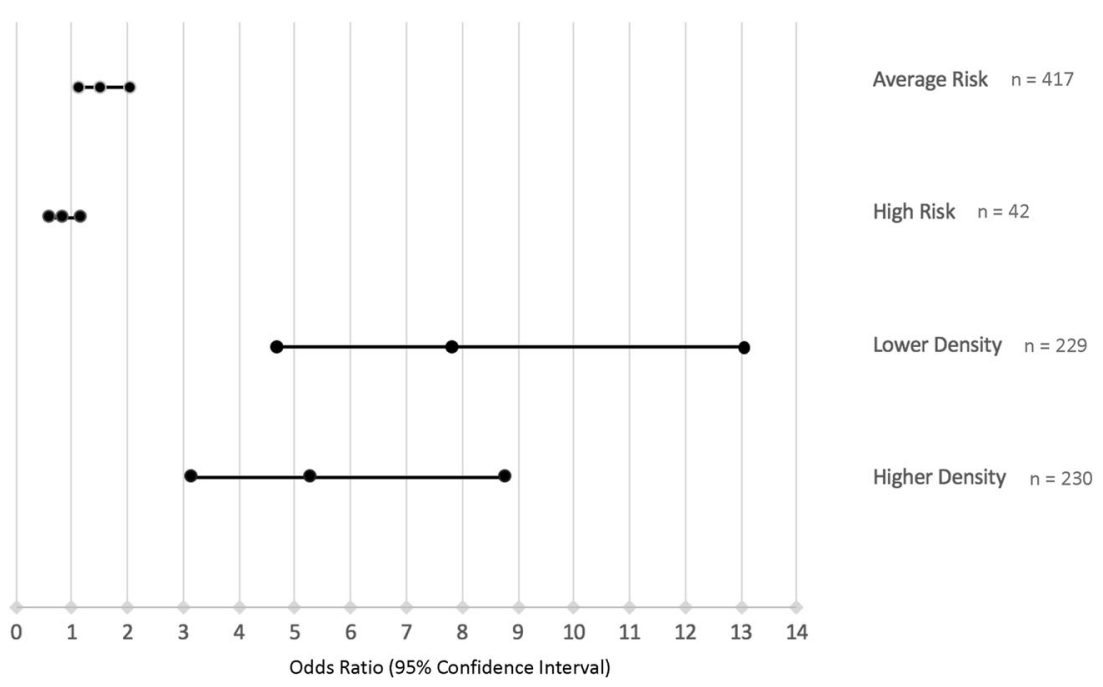

Figure 3 Effect of the intervention on knowledge of calculated breast cancer risk and measured breast density by breast cancer risk and breast density. 
Table 2 Discussion of the Mammogram Result

\begin{tabular}{|c|c|c|c|}
\hline & Intervention & Control & $p$ value \\
\hline $\begin{array}{l}\text { Discussed results of } \\
\text { mammogram with primary } \\
\text { care provider } \\
\text { If yes, discussed: }\end{array}$ & $78(33.5 \%)$ & $\begin{array}{l}56 \\
(25.5 \%)\end{array}$ & .05 \\
\hline $\begin{array}{l}\text { Whether you should get any } \\
\text { breast cancer in addition to a } \\
\text { mammogram }\end{array}$ & $28(35.9)$ & $\begin{array}{l}12 \\
(21.4)\end{array}$ & .07 \\
\hline Ultrasound & $14(18.0)$ & $3(5.4)$ & .03 \\
\hline Breast MRI & $11(14.4)$ & $7(12.5)$ & .79 \\
\hline
\end{tabular}

*p value calculated using chi-square test

\section{Use of and Satisfaction with the Intervention}

Among women in the intervention arm, $86 \%$ watched the entire video, $4 \%$ part of the video, and $10 \%$ did not watch the video. Videos were well received with $86 \%$ of respondents saying that they were very easy to understand.

\section{DISCUSSION}

The evidence base to inform the implementation of MBD legislation is complex, incomplete, and controversial, ${ }^{15}$ and tools for patient education are limited. INFORMD demonstrates that brief, personalized videos can be implemented in clinical practice to improve personal knowledge of MBD and, to a smaller extent, personal risk of breast cancer, compared to a legislatively required letter. Since knowledge of risk was relatively high in this population at baseline, it is not surprising that an improvement in knowledge of risk associated with the intervention was smaller than that for MBD. We also demonstrated a modest improvement of discussion of these issues with the patient's PCP; this is not surprising given the short follow-up time interval as mammograms typically occur dissynchronously from PCP visits. Among those who talked with their PCP, the intervention was associated with greater discussion of supplemental ultrasound but not MRI. The video format was well received.

There is limited information about whether MBD legislation has promoted patient knowledge. A national, internet panel survey of over 2000 women in 2012 found that women residing in Connecticut were more likely to have discussed their MBD with a health care provider $(67 \%$ vs. $43 \%$ for residents of other US states). ${ }^{16}$ Eligibility for this crosssectional survey did not require recent mammography and did not assess personal knowledge of MBD or risk. To fill a need created by the legislation, providers want tools to help promote informed decision-making about MBD and breast cancer screening. ${ }^{10}$ While several studies have examined decision tools to systematically identify breast cancer risk in clinical settings and to increase patient-PCP discussion of breast cancer risk, ${ }^{14,17,18}$ we do now know of studies to date that have specifically examined the role of MBD notification on decision-making, although studies are underway. ${ }^{19}$
Our study has several limitations. We used the BCSC model as it incorporates information about density into the risk assessment, has been externally validated, and performs well across risk factor subgroups, ${ }^{12}$ but it does not assess genetic or lifestyle risk factors. We chose a threshold of 5\% to defined "higher risk" vs. the $3 \%$ threshold that is suggested by the US Preventive Services Task Force for consideration of the use of selective estrogen receptor modulators (SERMs) because our goal was to encourage women to discuss their risk of screening in addition to the specific use of SERMS. ${ }^{20}$ Further efforts are needed to ensure that these types of tools are generalizable to non-English speaking, women who do not have access to the internet, and women with less education from a variety of institutions. Our follow-up period was brief; longer-term studies are needed to understand the effect of these educational tools on knowledge of risk and MBD and screening utilization over longer time periods. We did not assess why women in the intervention arm were more likely to discuss their results with their PCP.

In summary, INFORMD demonstrated that a brief, personalized video following mammography can improve knowledge of personal MBD and risk of breast cancer compared to a legislatively mandated informational letter. Legislatively required language alone was not associated with improvement in knowledge. The videos were implemented in clinical practice without disruption of work flow and were well accepted by patients.

Acknowledgements: We would like to acknowledge Debora Kim, Center for Population Health Patient Engagement at Brigham and Women's Hospital, for her help filming the Vidscrip. This study was supported by a grant from the Controlled Risk Insurance Company, Ltd. (CRICO)/ Risk Management Foundation of the Harvard Medical Institutions; the funder did not have a role in the design or conduct of the study.

Corresponding Author: Jennifer S. Haas, MD, MSc; Division of General Medicine and Primary Care, Department of Medicine Brigham and Women's Hospital, Boston, MA, USA (e-mail: jhaas@bwh. harvard.edu).

\section{Compliance with Ethical Standards}

The protocol was reviewed and approved by the Institutional Review Board (IRB) of Partners Healthcare.

Conflict of Interest: The authors declare that they do not have a conflict of interest.

\section{REFERENCES}

1. Smith RA, Kerlikowske K, Miglioretti DL, Kalager M. Clinical decisions. Mammography screening for breast cancer. N Engl J Med 2012;367:e31.

2. Kerlikowske K, Zhu W, Tosteson AN, Sprague BL, Tice JA, Lehman CD, Miglioretti DL, Breast Cancer Surveillance C. Identifying women with dense breasts at high risk for interval cancer: a cohort study. Ann Intern Med 2015; 162:673-81.

3. Porter GJ, Evans AJ, Cornford EJ, Burrell HC, James JJ, Lee AH, Chakrabarti J. Influence of mammographic parenchymal pattern in screening-detected and interval invasive breast cancers on pathologic features, mammographic features, and patient survival. AJR Am J Roentgenol 2007;188:676-83. 
4. Kerlikowske K, Zhu W, Hubbard RA, Geller B, Dittus K, Braithwaite D, Wernli KJ, Miglioretti DL, O'Meara ES. Outcomes of Screening Mammography by Frequency, Breast Density, and Postmenopausal Hormone Therapy. JAMA Intern Med 2013:1-10.

5. Are You Dense? Advocacy Because Your Life Matters. Are You Dense Advocacy, Inc., 2015. (Accessed July 11, 2018, at http:// areyoudenseadvocacy.org/.)

6. Massachusetts Breast Density Notification Law. 2015. (Accessed July 11, 2018, at http://www.massmed.org/Patient-Care/MassachusettsBreast-Density-Notification-Law/\#.WnnCVbynFhE.)

7. Sprague BL, Gangnon RE, Burt V, Trentham-Dietz A, Hampton JM, Wellman RD, Kerlikowske $\mathbf{K}$, Miglioretti DL. Prevalence of mammographically dense breasts in the United States. J Natl Cancer Inst 2014; 106 .

8. Kressin NR, Gunn CM, Battaglia TA. Content, Readability, and Understandability of Dense Breast Notifications by State. JAMA 2016;315:1786-8.

9. Santiago-Rivas M, Benjamin S, Jandorf L. Breast Density Knowledge and Awareness: A Review of Literature. J Prim Care Community Health 2016;7:207-14

10. Klinger EV, Kaplan CP, St. Hubert S, Birdwell RL, Haas JS. Patient and Provider Perspectives on Mammographic Breast Density Notification Legislation. MDM Policy \& Practice 2017;1:1-8.

11. Tice JA, Cummings SR, Ziv E, Kerlikowske K. Mammographic breast density and the Gail model for breast cancer risk prediction in a screening population. Breast Cancer Res Treat 2005;94:115-22.

12. Tice JA, Cummings SR, Smith-Bindman R, Ichikawa L, Barlow WE, Kerlikowske $\mathbf{K}$. Using clinical factors and mammographic breast density to estimate breast cancer risk: development and validation of a new predictive model. Ann Intern Med 2008;148:337-47.
13. Tice JA, Miglioretti DL, Li CS, Vachon CM, Gard CC, Kerlikowske K. Breast Density and Benign Breast Disease: Risk Assessment to Identify Women at High Risk of Breast Cancer. J Clin Oncol 2015;33:3137-43.

14. Kaplan CP, Livaudais-Toman J, Tice JA, Kerlikowske K, Gregorich SE, Perez-Stable EJ, Pasick RJ, Chen A, Quinn J, Karliner LS. A randomized, controlled trial to increase discussion of breast cancer in primary care. Cancer Epidemiol Biomark Prev 2014;23:1245-53.

15. Haas JS, Kaplan CP. The Divide Between Breast Density Notification Laws and Evidence-Based Guidelines for Breast Cancer Screening: Legislating Practice. JAMA Intern Med 2015;175:1439-40.

16. Rhodes DJ, Radecki Breitkopf C, Ziegenfuss JY, Jenkins SM, Vachon CM. Awareness of breast density and its impact on breast cancer detection and risk. J Clin Oncol Off $\mathrm{J}$ Am Soc Clin Oncol 2015;33: 1143-50.

17. Schonberg MA, Hamel MB, Davis RB, Griggs MC, Wee CC, Fagerlin A Marcantonio ER. Development and evaluation of a decision aid on mammography screening for women 75 years and older. JAMA Intern Med 2014;174:417-24.

18. Haas JS, Baer HJ, Eibensteiner K, Klinger EV, St Hubert S, Getty G Brawarsky P, Orav EJ, Onega T, Tosteson AN, Bates DW, Colditz G. A Cluster Randomized Trial of a Personalized Multi-Condition Risk Assessment in Primary Care. Am J Prev Med 2017;52:100-5.

19. Knerr S, Wernli KJ, Leppig K, Ehrlich K, Graham AL, Farrell D, Evans C, Luta G, Schwartz MD, O'Neill SC. A web-based personalized risk communication and decision-making tool for women with dense breasts: Design and methods of a randomized controlled trial within an integrated health care system. Contemp Clin Trials 2017;56:25-33.

20. Moyer VA, for the US Preventive Services Task Force. Medications to decrease the risk for breast cancer in women: recommendations from the U.S. Preventive Services Task Force recommendation statement. Ann Intern Med 2013;159:698-708. 